\title{
Genomic characterization of clear cell renal cell carcinoma using targeted gene sequencing
}

\author{
PO-HUNG LIN ${ }^{1,2 *}$, CHAO-YUAN HUANG ${ }^{3 *}$, KAI-JIE YU ${ }^{1}$, HUNG-CHENG KAN $^{1}$, CHUNG-YI LIU $^{1}$, \\ CHENG-KENG CHUANG ${ }^{1}$, YU-CHUAN LU ${ }^{3,4}$, YING-HSU CHANG ${ }^{1}$, I-HUNG SHAO ${ }^{1}$ and SEE-TONG PANG ${ }^{1}$ \\ ${ }^{1}$ Division of Urology, Department of Surgery, Chang Gung Memorial Hospital at Linkou; ${ }^{2}$ Graduate Institute of Clinical \\ Medical Science, College of Medicine, Chang Gung University, Taoyuan 333; ${ }^{3}$ Department of Urology, \\ National Taiwan University Hospital; ${ }^{4}$ Institute of Biomedical Engineering, \\ National Taiwan University, Taipei 10022, Taiwan, R.O.C.
}

Received May 4, 2020; Accepted November 10, 2020

DOI: $10.3892 / \mathrm{ol} .2021 .12430$

\begin{abstract}
Kidney cancer is one of the most lethal cancer types worldwide. The most common subtype of kidney cancer is clear cell renal cell carcinoma (ccRCC), and the somatic mutations of ccRCC have been identified through the development of large databases. The present study aimed to validate the status of the associated gene mutations in a Taiwanese cohort. Targeted sequencing was used to validate the mutation status of genes related to ccRCC in Taiwanese patients who had nephrectomy for kidney cancer. The top eight mutated genes in the Catalogue Of Somatic Mutations In Cancer (COSMIC) were selected. These genes were VHL, protein polybromo-1 (PBRM1), histone-lysine N-methyltransferase SETD2, BRCA1-associated protein-1 $(B A P 1)$, lysine-specific demethylase 5C (KDM5C), TP53, MTOR and PTEN. The association between the gene mutation status of VHL, PBRM1, SETD2 and BAP1 was validated with clinicopathological parameters as well as overall survival time. Tumor cells from 96 patients with ccRCC were target sequenced. The order of mutation rate of the eight aforementioned genes was similar to that reported within COSMIC. The present Taiwanese cohort exhibited lower PBRM1 and $B A P 1$ mutation rates compared with average, with increased mutation rates for SETD2 and KDM5C. BAP1 mutation was associated with the tumor and cancerous stage. None of these four genes were positively associated with the overall survival of patients. The PBRM1 and SETD2 mutations were mutually exclusive to $B A P 1$ mutation. Overall, the present study provided
\end{abstract}

Correspondence to: Dr See-Tong Pang, Division of Urology, Department of Surgery, Chang Gung Memorial Hospital at Linkou, 5 Fuxing Street, Taoyuan 333, Taiwan, R.O.C.

E-mail: pst64lab@gmail.com

${ }^{*}$ Contributed equally

Key words: clear cell renal cell carcinoma, targeted gene sequencing, VHL, protein polybromo-1, SETD2, BRCA1-associated protein-1 data confirming gene alteration in Taiwanese patients with ccRCC and showed some differences when compared with Western countries. Further comprehensive genomic and epigenomic studies, as well as downstream validation, are necessary to evaluate the impact of these differences.

\section{Introduction}

Kidney cancer is one of the most lethal cancer types globally. In the United States, the 5-year survival rate of metastatic kidney cancer was $\sim 12 \%$ between 2014 and 2018 (1). The estimated number of newly diagnosed kidney cancer cases annually is 73,820 , and the projected number of kidney cancer-associated deaths in 2019 in the United States was 14,770 (1). In Taiwan, in 2016, there were 1,364 newly diagnosed kidney cancer cases, and 600 patients died of kidney cancer (2). The incidence rate of renal cell carcinoma ( $\mathrm{RCC}$ ) in males and females in Taiwan in 2016 was 7.75 and 3.86 per 100,000 population, respectively, compared with 22.2 and 11.4 per 100,000 population, respectively, in the US $(1,2)$. Although the incidence of RCC in Taiwan is not as high as that in Western countries, it is still an important public health issue affecting patients with a median age of diagnosis 61 and 62 years old in males and females, respectively (2).

Clear cell RCC (ccRCC) is the most common histological subtype of kidney cancer, which accounts for around 70-75\% of all renal malignancies globally (3). The most distinct mutated driver gene of the ccRCC is the VHL gene, which is found to be mutated in $51 \%$ of all patients with ccRCC globally (4). On the basis of the investigation of the VHL pathway, the tyrosine kinase inhibitors (TKIs) that target this pathway were established as the mainstay for systemic therapy for metastatic ccRCC since the early 21 st century (5). The VHL gene encodes the VHL protein, an E3 ubiquitin ligase, which targets the hypoxia inducible factors. One such example is hypoxia inducible factor- $1 \alpha$ (HIF-1 $\alpha)$, which is the most researched target. When $V H L$ is mutated, HIF-1 $\alpha$ cannot be degraded and accumulates, inducing the expression of several angiogenesis-related factors, such as vascular endothelial growth factor, platelet-derived growth factor (PDGF) and TGF- $\beta$. This is an important process of tumorigenesis of RCC (6). TKIs block the pathways of angiogenesis and therefore inhibit tumor 
growth. Keeping this in mind, it is important to understand the mutations underpinning the pathogenesis of any cancer not only for diagnosis but also for treatment.

Through the high throughput sequencing methods developed in recent decades, gene alteration databases have been developed within large-scale projects, such as The Cancer Genome Atlas (TCGA) and Catalogue Of Somatic Mutations In Cancer (COSMIC) (4). In addition to VHL, the top ten mutated genes in ccRCC are the following: Protein polybromo-1 (PBRM1), histone-lysine N-methyltransferase SETD2, BRCA1-associated protein-1 (BAPl), lysine-specific demethylase 5C (KDM5C), TP53, MTOR, PTEN, low-density lipoprotein receptor-related protein $1 \mathrm{~B}$ and Lysine $\mathrm{N}$-methyltransferase 2C (4). Numerous epigenomic-related genes are mutated in ccRCC, which suggests that epigenetic regulation plays an important role in the molecular pathways underpinning ccRCC, hence leading to the development of possible epigenetic therapies (7). However, most of the candidate genes in these databases are based on Western populations. For example, only seven (1.9\%) Asian patients were included in TCGA database (8). Comparing somatic mutations in kidney cancer between patients in Asia and Western countries is necessary since the incidence rate is different and there may be some possible interethnic genetic differences.

In the present study, targeted gene sequencing was used to evaluate gene alteration(s) in Taiwanese patients with ccRCC. The top eight mutated genes in the COSMIC database were targeted and the association between their gene mutation status and clinical and pathological parameters and survival outcome of patients was determined.

\section{Material and methods}

Patients. Patients were enrolled from the Chang Gung Memorial (Taoyuan, Taiwan R.O.C.) between January 2006 and December 2010 and National Taiwan University Hospital (Taipei, Taiwan R.O.C.) between Jan 1st 2013 and Dec 31st 2014. The patients enrolled in the study were subjective to the willing of participating and the availability of tissue samples. The inclusion criteria of the study were as follows: i) Patients who received radical/partial nephrectomy, ii) Pathology diagnosis of clear cell RCC and iii) Willing to participate and provided signed informed consent. The exclusion criteria were: i) Histology types other than clear cell RCC and ii) No adequate specimen available. All patients with a renal tumor diagnosis received either partial or radical nephrectomy according to clinical indications. The pathology of each tumor was reviewed by pathologists specializing in kidney cancer identification, and only those diagnosed as ccRCC were included in the study. We randomly selected 96 patients with ccRCC for this study. Clinical demographic parameters, cancer stage using the American Joint Committee on Cancer (9) and pathological data including tumor stage, lymph node status and Fuhrman grade were collected. The overall survival time was determined as from the date of operation to the date of death. If there was no date of death, the data would be censored using the last date of follow-up at the outpatient department.

Ethical statements. The study was approved by the Institutional Review Board of Chang Gung Memorial Hospital (approval no. 106-3050C) and National Taiwan University Hospital (approval no. 201312158RIND). The retrospective genetic study and the treatment plan for the patients was conducted according to clinical guidelines and standard of care. The present genetic study results did not affect the treatment plan of patients following surgery. Informed written consent was provided by all patients.

Sample collection. After removal of the tumor, a specimen without necrosis of $\sim 5 \mathrm{~mm}^{3}$ at the central area of the tumor was excised and packed in foil. Then, the samples were stored liquid nitrogen tank $\left(-196^{\circ} \mathrm{C}\right)$ within $1 \mathrm{~h}$ of collection. All procedures were performed under aseptic conditions.

DNA extraction. DNA was extracted from the aforementioned samples using the Qiagen blood and tissue DNeasy Blood \& Tissue extraction kit (Qiagen $\mathrm{GmbH}$ ) according to the manufacturer's protocol. Briefly, $\sim 25 \mathrm{mg}$ of tumor tissue was minced and transferred to a $1.5-\mathrm{ml}$ microcentrifuge tube, and $180 \mu \mathrm{l}$ ATL buffer and $20 \mu \mathrm{l}$ proteinase $\mathrm{K}$ were added (all included in the aforementioned kit). The tube was incubated at $56^{\circ} \mathrm{C}$ until totally lysed, then $4 \mu 1$ RNase A was added, and the sample was incubated for $2 \mathrm{~min}$ at room temperature. After vortexing, $200 \mu \mathrm{l}$ AL buffer was added, followed by mixing and addition of $200 \mathrm{ml}$ absolute ethanol. The sample was transferred to a DNeasy Mini spin column and centrifuged at 7,000 $\mathrm{xg}$ at room temperature for $1 \mathrm{~min}$, followed by washing and elution of DNA in nuclease-free water. The nucleic acid concentration was measured with NanoDrop 1000 (Thermo Fisher Scientific, Inc.), and $1 \%$ agarose gel electrophoresis with ethidium bromide illumination was performed for quality control.

Targeted genes. To compare gene alterations between Taiwanese patients and patients in the COSMIC and TCGA databases, the top eight most frequently mutated genes of ccRCC in the COSMIC database were investigated (VHL, PBRM1, SETD2, BAP1, TP53, KDM5C, MTOR and PTEN). A multiplex PCR target enrichment panel for target-relevant genes was enriched with DNA GeneRead DNAseq Custom panel V2 (cat. no. 181902 CNGHS-02735X-67). The DNA panel was designed using the Qiagen GeneRead designer website (https://www.qiagen.com/us/shop/genes-and-pathways/custom-products/custom-array-products/generead-designer/).

Library preparation and targeted gene sequencing. Targeted sequencing was performed according to a previously described protocol (10). Briefly, DNA libraries were prepared using components from TruSeq DNA Sample Preparation kits (Illumina, Inc.). For each sample, 80 ng DNA was used as starting material. The DNA was enzymatically fragmented and end-repaired, and the reaction was carried out at $4^{\circ} \mathrm{C}$ for $1 \mathrm{~min}, 32^{\circ} \mathrm{C}$ for $24 \mathrm{~min}$ and $65^{\circ} \mathrm{C}$ for $30 \mathrm{~min}$. Immediately after the reaction, ligation of barcoded adapters was performed, and the reaction continued at $20^{\circ} \mathrm{C}$ for $15 \mathrm{~min}$. Purification was carried out to remove the free barcoded adapters, with subsequent PCR enrichment for the targeted genes under the following conditions: $95^{\circ} \mathrm{C}$ For $13 \mathrm{~min}, 98^{\circ} \mathrm{C}$ for $2 \mathrm{~min}$, then six cycles of $98^{\circ} \mathrm{C}$ for $15 \mathrm{sec}$ and $65^{\circ} \mathrm{C}$ for $15 \mathrm{~min}$, and finally $72^{\circ} \mathrm{C}$ for $5 \mathrm{~min}$. Each reaction was cleaned up using 0.9x Ampure beads (Beckman Coulter, Inc.) to remove unbound primers. 
For library preparation, the NEBNext Multiplex Oligos kit was used (New England BioLabs, Inc.). The enriched DNA was combined with universal primers, identical index primers and a PCR master mix supplied in the kit. The universal PCR conditions were as follows: $95^{\circ} \mathrm{C}$ For $13 \mathrm{~min}, 98^{\circ} \mathrm{C}$ for $2 \mathrm{~min}$, 20 cycles of $98^{\circ} \mathrm{C}$ for $15 \mathrm{sec}$ and $60^{\circ} \mathrm{C}$ for $2 \mathrm{~min}$, and $72^{\circ} \mathrm{C}$ for $5 \mathrm{~min}$. Gel electrophoresis was performed to ensure that the fragmental DNA library length was between 400 and 500 base pairs, and the appropriate band was excised and purified using the QIAquick Gel Extraction kit (Qiagen $\mathrm{GmbH}$ ). All libraries were sequenced on an Illumina MiSeq sequencer (pair-end, 2 x $300 \mathrm{bp}$ ) following the manufacturer's instructions (Illumina, Inc.).

Data processing and analysis. The smCounter was used to generate data as previously described (10). At each target locus, posterior probabilities of the alleles (including possible indels) were first calculated on the barcode level, noted as $P$ (AllelelBC $k$ ) for the $k^{\text {th }}$ barcode. Assuming that the locus is covered by $\mathrm{N}$ mutually independent barcodes, a prediction index $I=-\sum_{k=1}^{N} \log _{10}[1-P($ AllelelBC $k)]$ is assigned to each allele, representing the likelihood that the allele exists in at least one DNA molecule. If a non-reference allele's prediction index exceeds the preselected threshold, this allele is considered a candidate variant. Candidate variants were confirmed only if they passed all of the post-processing filters. The analysis process was as follows: i) Raw reads QC as adapter trimming and quality filtering, ii) Reference alignment using the Burrows-Wheeler Transform algorithm (11), iii) Variant calling using the Genome Analysis Toolkit (12), iv) Somatic mutation detection using Mutect (13) and v) Variant annotation using VEP (14).

Sanger sequencing validation. Sanger sequencing was used to validate the mutated genes uncovered within the targeted sequencing data. The primers were designed using Primer3 software (http://frodo.wi.mit.edu). Purified PCR products were sequenced in both forward and reverse directions using ABI PRISM BigDye Terminator Cycle Sequencing Ready Reaction kits (version 3; Applied Biosystems; Thermo Fisher Scientific, Inc.) and an ABI PRISM 3730 Genetic Analyzer (Thermo Fisher Scientific, Inc.).

Statistics. The $\chi^{2}$ test was used to validate the association between mutated genes and clinicopathological parameters. Data are presented as mean \pm standard deviation. Fisher's exact test was used to validate the relationship between factors with sample sizes less than five. A Kaplan-Meier log-rank test model was used to evaluate the relationship between mutated genes and survival of patients. $\mathrm{P}<0.05$ was considered to indicate a statistically significant difference. There was no repeat for the targeted sequencing and every sample was sequenced once only. All statistics were performed using SPSS version 22 (IBM Corp).

Genetic database comparison. The mutational percentage of the eight targeted-sequenced genes were compared to the data from the COSMIC and TCGA databases. To access the COSMIC database, the following search terms were used: 'Kidney' in the tissue selection section, 'include all' in the sub-tissue selection section, 'carcinoma' in the histology selection section and 'clear cell renal cell carcinoma' in the sub-histology selection section. The top 20 genes were reported. The top mutated genes of kidney cancer in TCGA database were previously published (8), so the COSMIC and TCGA results were compared directly.

\section{Results}

Demographic data and clinicopathological parameters. A total of 96 patients with sporadic kidney cancer who fulfilled the inclusion criteria were randomly selected for the present study. The operations and sample collection were performed between 2006 and 2014. The mean follow-up time was $39.42 \pm 29.85$ months (range, $1-124$ months). In total, 12 patients $(12.5 \%)$ died during follow-up. A summary of the demographic data is shown in Table I. Among 96 patients, 64.6 and 34.4\% were male and female, respectively. The mean age at diagnosis was $57.64 \pm 14.73$ years old (data not shown). Approximately $72 \%$ of patients received radical nephrectomy. A tumor size $>4 \mathrm{~cm}$ accounted for $71.9 \%$ of all included tumors. This was compatible with the percentage of patients having received radical nephrectomy, as partial nephrectomy is usually performed for patients with T1a renal tumors. Approximately two-thirds of patients exhibited localized disease (stages I and II), and locally advanced or metastatic disease (stages III and IV) occurred in $34.4 \%$ of patients. Twelves $(12.5 \%)$ patients had metastatic disease and received TKIs as systemic treatments.

Summary of somatic mutations. A total of 6,516 nonsynonymous mutations in exons and 565 mutations at splice junctions were observed within the 96 samples. Among the non-synonymous mutations, there were 5,908 missense mutations, 323 frameshift mutations, 11 in-frame deletions or insertions, and two start-loss and 278 stop-gain mutations. The most frequent single nucleotide substitution in missense mutations was T:A to G:C (data not shown). The percentage and type of gene mutation are shown in Fig. 1. There were three genes that exceeded $20 \%$ of the mutation rate: $V H L$, PBRM1 And SETD2. The somatic mutations mapped to genes are shown in Fig. 2.

Sanger sequencing validation. Sanger sequencing was used to validate the accuracy of targeted sequencing for the three targeted genes $V H L, P B R M 1$ and $B A P 1$ since we only sequenced the samples once. The consensus rates of each gene were 93.8, 93.3 and 100\% for $V H L, P B R M 1$ and $B A P 1$, respectively (data not shown). The high consensus rates indicated that the targeted sequencing data were reliable, and the mutations were true mutations. Some selected results of the Sanger sequencing were shown in the supplementary figures. Each panel in the supplementary figures indicated individual samples, which were labeled as NTCG or RCC followed by digits. Fig. S1 presents the selected results of Sanger sequencing validation for $V H L$ indels. Panels $(\mathrm{A})$ to $(\mathrm{H})$ reveal the position of mutation and the resulted frameshift mutation of associated amino acids. Fig. S2 shows the selected results of Sanger sequencing validation for VHL SNVs. Panels (A) to (C) show the position of mutation and the resulted associated 
Table I. Demographic and clinicopathological parameters and the association with gene mutation status.

\begin{tabular}{|c|c|c|c|c|c|c|c|c|c|c|c|c|c|}
\hline \multirow[b]{2}{*}{ Characteristic } & \multirow[b]{2}{*}{ Patient n (\%) } & \multicolumn{2}{|c|}{$\begin{array}{c}\begin{array}{c}V H L \\
\text { mutation }\end{array} \\
\end{array}$} & \multirow[b]{2}{*}{$\mathrm{P}$-value } & \multicolumn{2}{|c|}{$\begin{array}{l}\text { PBRM1 } \\
\text { mutation }\end{array}$} & \multirow[b]{2}{*}{ P-value } & \multicolumn{2}{|c|}{$\begin{array}{c}\text { SETD2 } \\
\text { mutation }\end{array}$} & \multirow[b]{2}{*}{ P-value } & \multicolumn{2}{|c|}{$\begin{array}{l}\text { BAP1 } \\
\text { mutation }\end{array}$} & \multirow[b]{2}{*}{ P-value } \\
\hline & & Yes & No & & Yes & No & & Yes & No & & Yes & No & \\
\hline Total & 96 & 48 & 48 & & 25 & 71 & & 21 & 75 & & 9 & 87 & \\
\hline Sex & & & & $0.010^{\mathrm{a}}$ & & & $0.007^{\mathrm{b}}$ & & & 0.120 & & & 1.000 \\
\hline Male & $62(64.6)$ & 37 & 25 & & 22 & 40 & & 4 & 30 & & 6 & 56 & \\
\hline Female & $34(35.4)$ & 11 & 23 & & 3 & 31 & & 17 & 45 & & 3 & 31 & \\
\hline Age, years & & & & 0.289 & & & $0.018^{\mathrm{a}}$ & & & $0.026^{\mathrm{a}}$ & & & 0.720 \\
\hline$<65$ & $61(63.5)$ & 28 & 33 & & 11 & 50 & & 9 & 52 & & 5 & 56 & \\
\hline$\geq 65$ & $35(36.5)$ & 20 & 15 & & 14 & 21 & & 12 & 23 & & 4 & 31 & \\
\hline Tumor location & & & & 0.525 & & & 0.307 & & & & & & 1.000 \\
\hline Right & $35(36.5)$ & 7 & 28 & & 7 & 28 & & 5 & 30 & 0.173 & 3 & 32 & \\
\hline Left & $61(63.5)$ & 18 & 43 & & 18 & 43 & & 16 & 45 & & 6 & 55 & \\
\hline Type of operation & & & & 0.418 & & & 0.441 & & & 0.169 & & & 0.102 \\
\hline Radical nephrectomy & 69 (71.9) & 33 & 36 & & 16 & 53 & & 12 & 57 & & 8 & 61 & \\
\hline Partial nephrectomy & $26(27.1)$ & 15 & 11 & & 9 & 17 & & 9 & 17 & & 0 & 26 & \\
\hline Missing data & 1 & 0 & 1 & & 0 & 1 & & 0 & 1 & & & & \\
\hline Tumor stage & & & & 0.681 & & & 0.101 & & & 0.314 & & & $0.007^{\mathrm{b}}$ \\
\hline $\mathrm{T} 1$ & $57(59.4)$ & 27 & 30 & & 16 & 41 & & 16 & 41 & & 1 & 56 & \\
\hline $\mathrm{T} 2$ & $10(10.4)$ & 4 & 6 & & 0 & 10 & & 1 & 9 & & 3 & 7 & \\
\hline $\mathrm{T} 3$ & $26(27.1)$ & 15 & 11 & & 7 & 19 & & 3 & 22 & & 4 & 22 & \\
\hline $\mathrm{T} 4$ & $3(3.1)$ & 2 & 1 & & 2 & 1 & & 1 & 3 & & 1 & 2 & \\
\hline Tumor size, $\mathrm{cm}$ & & & & 0.581 & & & 0.767 & & & 0.388 & & & $0.020^{\mathrm{a}}$ \\
\hline$\leq 4$ & $27(28.1)$ & 12 & 15 & & 7 & 20 & & 8 & 19 & & 0 & 27 & \\
\hline $4<$ size $\leq 7$ & $41(42.7)$ & 23 & 18 & & 12 & 29 & & 9 & 32 & & 3 & 38 & \\
\hline$>7$ & $28(29.2)$ & 13 & 15 & & 6 & 22 & & 4 & 24 & & 6 & 22 & \\
\hline TNM stage & & & & 0.628 & & & 0.286 & & & 0.296 & & & $0.012^{\mathrm{a}}$ \\
\hline I & $56(58.3)$ & 27 & 29 & & 16 & 40 & & 16 & 40 & & 1 & 55 & \\
\hline II & $7(7.3)$ & 3 & 4 & & 0 & 7 & & 1 & 6 & & 2 & 5 & \\
\hline III & $21(21.9)$ & 13 & 8 & & 7 & 14 & & 3 & 18 & & 3 & 18 & \\
\hline IV & $12(12.5)$ & 5 & 7 & & 2 & 10 & & 1 & 11 & & 3 & 9 & \\
\hline Fuhrman grade & & & & 0.505 & & & 0.085 & & & 0.876 & & & 0.233 \\
\hline 1 & $9(9.4)$ & 3 & 6 & & 0 & 9 & & 2 & 7 & & 0 & 9 & \\
\hline 2 & $47(49)$ & 25 & 22 & & 17 & 30 & & 12 & 35 & & 3 & 44 & \\
\hline 3 & $27(28.1)$ & 15 & 12 & & 7 & 20 & & 4 & 23 & & 5 & 22 & \\
\hline 4 & $8(8.3)$ & 4 & 4 & & 1 & 7 & & 2 & 6 & & 0 & 8 & \\
\hline No grade & $5(5.2)$ & 1 & 4 & & 0 & 5 & & 1 & 4 & & 1 & 4 & \\
\hline
\end{tabular}

${ }^{\mathrm{a}} \mathrm{P}<0.05,{ }^{\mathrm{b}} \mathrm{P}<0.01$.

amino acid changes. Fig. S3 presents selected results of Sanger sequencing validation for PBRMI indels. Panel (A) to (I) reveal the position of mutation and the resulted frameshift mutation of associated amino acids. Fig. S4 shows the selected results of Sanger sequencing validation for PBRM1 SNVs. Panels (A) to $(\mathrm{E})$ show the position of mutation and the resulted associated amino acid changes. Fig. S5 reveals the selected results of Sanger sequencing validation for $B A P 1$ indels. Panels (A) to (C) reveal the position of mutation and the resulted frameshift mutation of associated amino acids.
Gene alteration in the Taiwanese cohort. The most frequently mutated gene was VHL (50\%), followed by PBRM1 (26\%) and SETD2 (22\%) (Fig. 1). Concurrence of VHL and PBRM1 mutations was found in $19(19.79 \%)$ patients in our cohort. Only one (1.04\%) patient had both $P B R M 1$ and $B A P 1$ mutations. None of the 96 patients had both SETD2 and BAP1 mutation (data not shown). The comparison of the gene alterations between the Taiwanese cohort and COSMIC/TCGA databases illustrated in Fig. 3 shows that the order of the mutational frequency between these cohorts was similar. However, the Taiwanese 


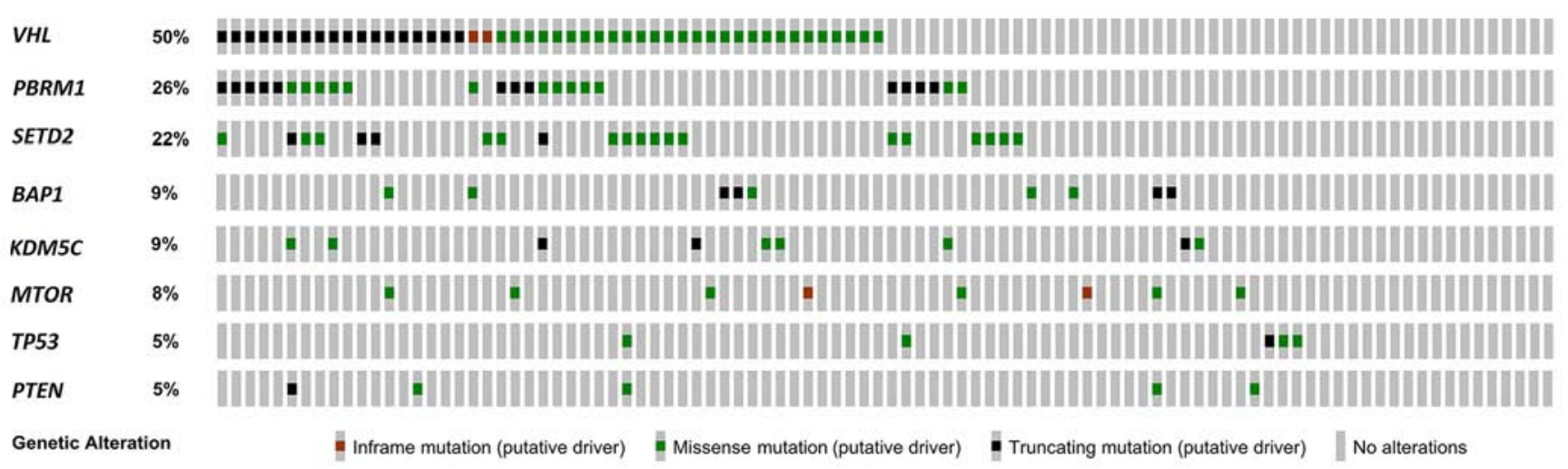

Figure 1. Alteration variants in each gene. Numbers indicate the percentage of gene mutation in 96 patients with clear cell renal cell carcinoma. PBRM1, protein polybromo-1; BAP1, BRCA1-associated protein-1; KDM5C, lysine-specific demethylase 5C.
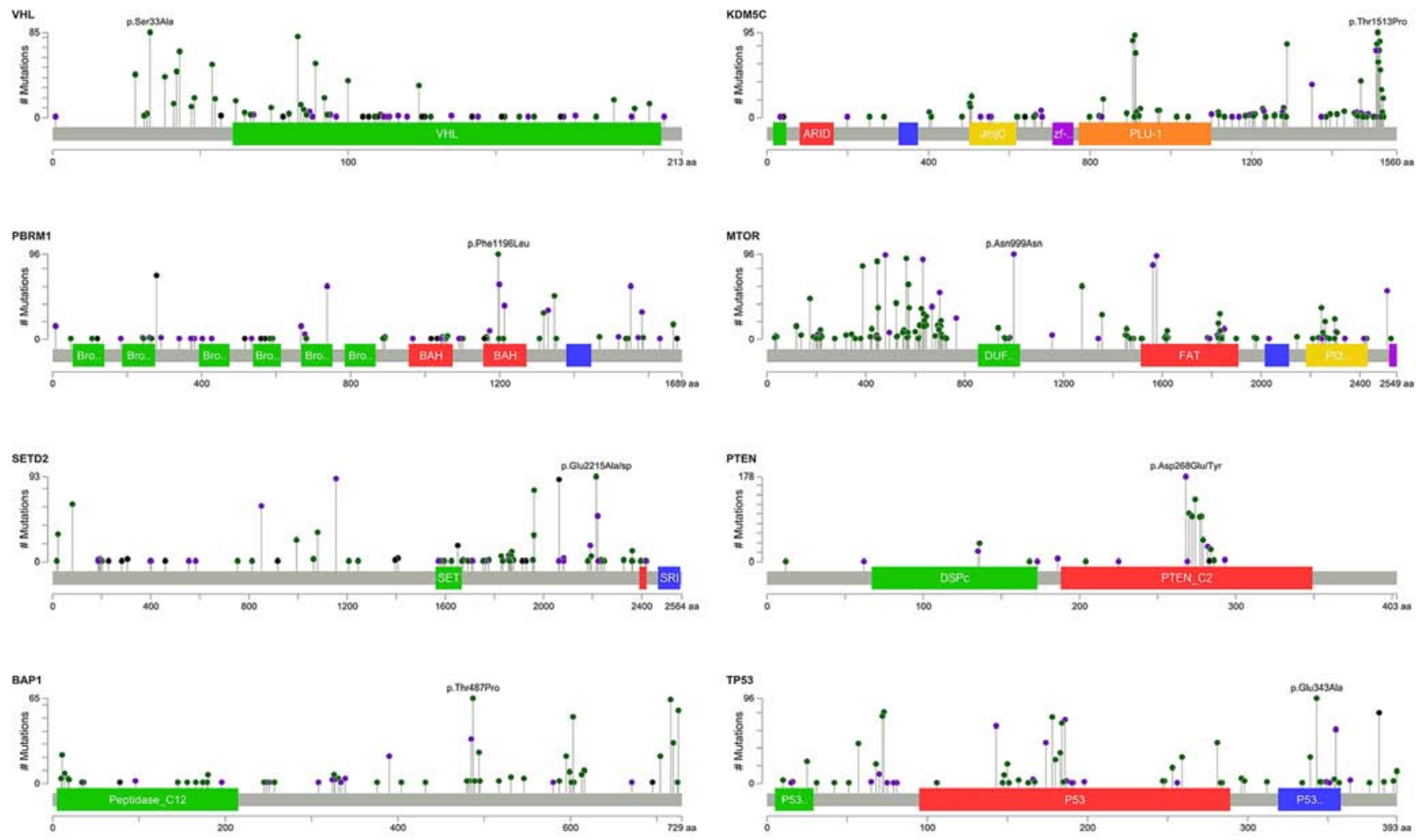

Figure 2. Mutation diagram of each gene with all somatic mutations shown. Green, purple, and black circles indicate missense, truncating and other mutations, respectively. PBRM1, protein polybromo-1; BAP1, BRCA1-associated protein-1; KDM5C, lysine-specific demethylase 5C.

cohort had lower mutation rates in PBRM1 (26 vs. 33/33\%) and $B A P 1$ (9 vs. $13 / 10 \%)$ and higher mutation rates in SETD2 (22 vs. 13/12\%) and KDM5C (9 vs. 7/7\%).

Gene alteration and clinicopathological parameters. The association between the mutation statuses of $V H L, P B R M 1$, SETD2 and BAPI and clinicopathological parameters were determined (Table I). Patient sex was significantly associated with mutations in VHL $(\mathrm{P}=0.01)$ and PBRM1 $(\mathrm{P}=0.007)$, with higher frequencies of mutations in each gene in males. Age was significantly associated with mutations in PBRMI $(\mathrm{P}=0.018)$ and SETD2 $(\mathrm{P}=0.026)$, with higher mutation rates in patients $\geq 65$ years old. The $B A P l$ mutation status was significantly different between the tumor size $(\mathrm{P}=0.020)$, tumor stage $(\mathrm{P}=0.007)$ and Tumor-Node-Metastasis stage $(\mathrm{P}=0.012)$. None of these top four genes were associated with the Fuhrman grade of the tumors. The association between the mutation status and survival of patients was validated for VHL, PBRM1, SETD2 and BAP1. There was no significant association between patient survival and mutational status of these four genes (Fig. 4).

\section{Discussion}

Kidney cancer incidence varies around the world and is highest in Northern America, Europe, Australia and New Zealand, 


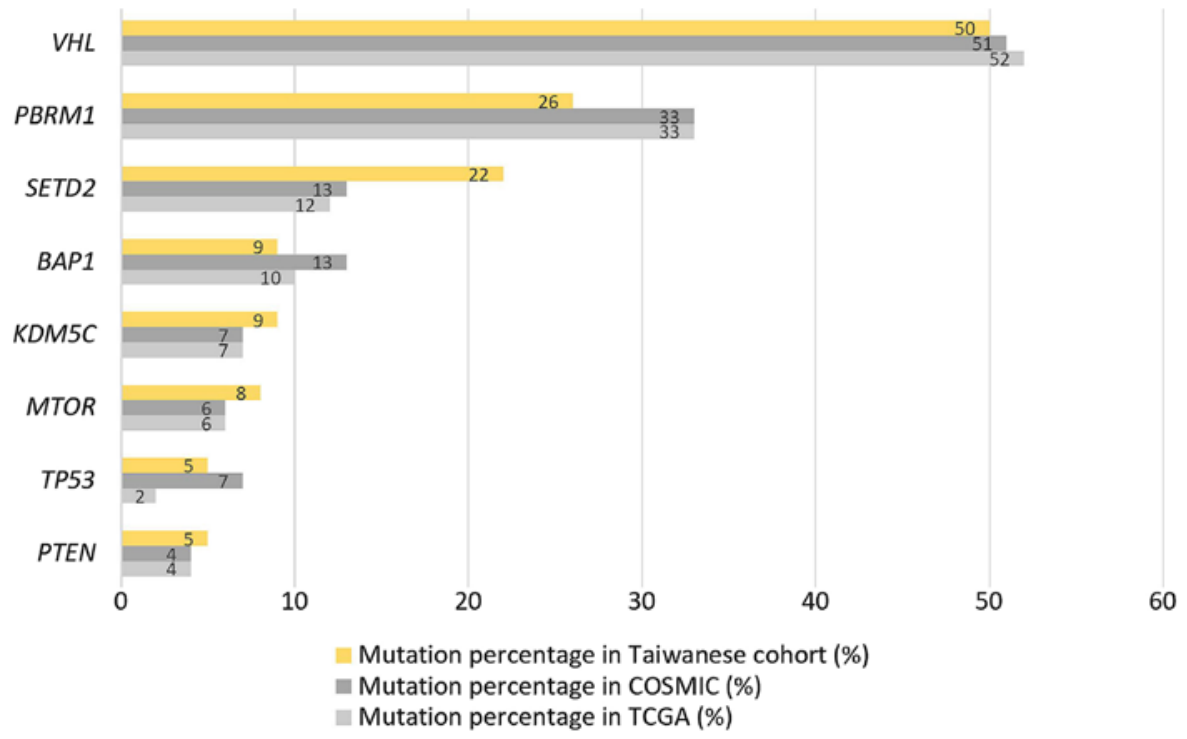

Figure 3. Comparison of gene mutation rates between the Taiwanese cohort and COSMIC/TCGA database. COSMIC, Catalogue Of Somatic Mutations In Cancer; TCGA, The Cancer Genome Atlas; PBRM1, protein polybromo-1; BAP1, BRCA1-associated protein-1; KDM5C, lysine-specific demethylase 5C.
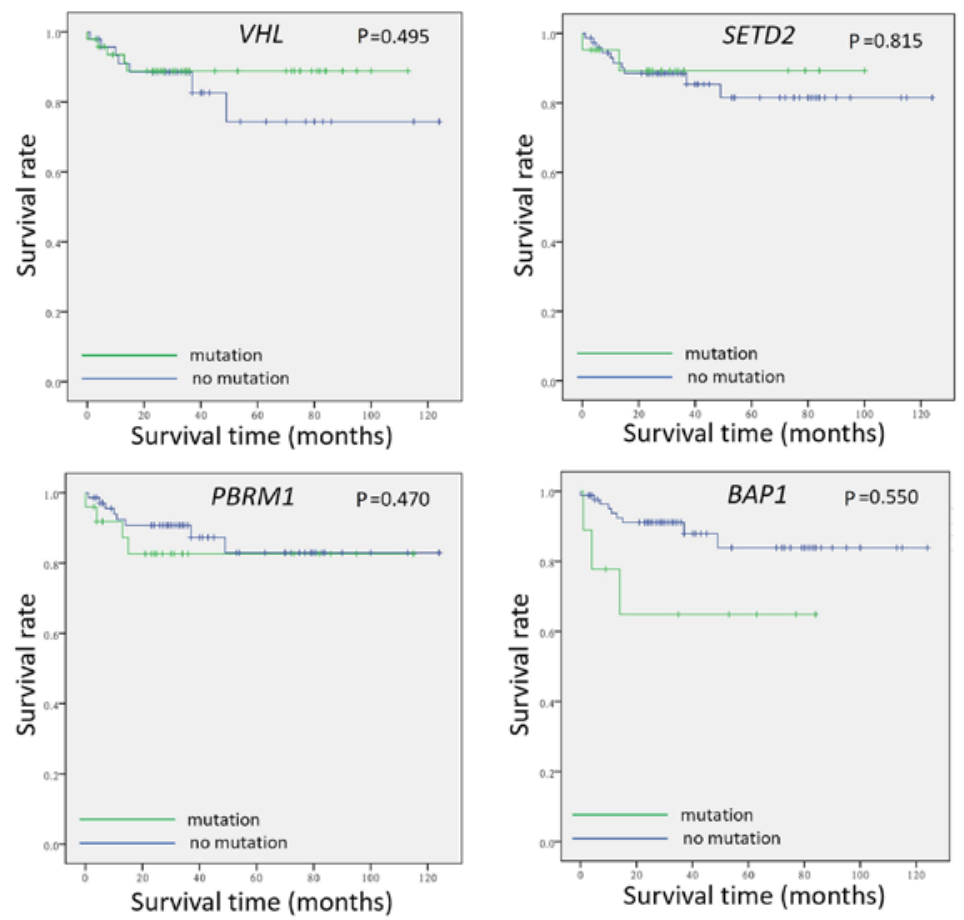

Figure 4. Association of gene mutation status and overall patient survival. PBRM1, protein polybromo-1; BAP1, BRCA1-associated protein-1.

with lower incidences in Asia and Africa (15). These differences in incidence may reflect varying diets and lifestyle, and interethnic genetic profiling may play a role as well. The present study demonstrated that the top eight most frequently mutated genes of ccRCC in COSMIC and Taiwan are similar, except for some differences in the mutation rate of particular genes. The analysis was focused on the top four genes $V H L$, PBRM1, SETD2 and BAP1, which are all located on chromosome $3 p$ (16). Chromosome $3 p$ deletion is frequent in ccRCC, resulting in high mutation rates of these genes (16). VHL was still the most frequently mutated gene, with lower mutation rates of $P B R M 1$ and $B A P I$ within the Taiwanese cohort and higher levels of SETD2 mutation compared with the COSMIC and TCGA databases.

The elevated mutation rate of VHL in the Taiwanese, TCGA and COSMIC cohorts suggested that the VHL pathway is the main pathogenic pathway in ccRCC globally. With normal oxygen levels and an intact $V H L$ gene, HIF- $1 \alpha$ binds to the VHL protein and is degraded via ubiquitylation. When $V H L$ is mutated, HIF-1 $\alpha$ accumulates and increases the transcription of genes containing the hypoxia response element (6). This would increase the expression of downstream proteins, such as the vascular endothelial growth factor, platelet-derived growth factor and transforming growth factor $\alpha$, thereby enhancing 
neoangiogenesis and carcinogenesis of ccRCC (17). Through the investigation of the role of the VHL pathway in the carcinogenesis of ccRCC, TKIs have become the mainstay of systemic treatments for metastatic ccRCC since the early 2000s (18). In a Japanese comprehensive mutational analysis study of the $V H L$ gene in patients with ccRCC, Kondo et al revealed that $V H L$ mutation is not associated with the clinicopathological parameters including tumor diameter, stage, grading, distant metastasis and lymph node metastasis. However, VHL is less frequently mutated in patients $>55$ years old (19). The present study also showed similar results, which suggested that there is no significant difference in the role of the VHL gene in ccRCC in Taiwanese patients.

$P B R M 1$ is the second most frequently mutated gene associated with ccRCC. PBRM1 encodes the BAF180 protein, which is a subunit of the SWI/SNF chromatin-remodeling complex (20). The SWI/SNF complex is a tumor suppressor, and mutations on the subunit-coding genes are found in numerous malignancies, such as lung, colorectal, pancreatic, head and neck and kidney cancer (20), especially in RCC (21). Nargund et al used a mouse model to show that the PBRM1 protein can inhibit the HIF1/STAT3 signaling pathway in $v h l^{-/}$cells. The loss of Pbrml function would position the mTORC1 activation at the third driver event of ccRCC (22). The present study provided evidence of sequential driver gene mutations in the pathogenesis of ccRCC. Concurrence of VHL and PBRM1 mutations was found in 19 (19.79\%) patients in our cohort.

The SETD2 gene encodes the SETD2 protein, a histone methyltransferase specific for lysine 36 located on histone H3 (H3K36). Methylation of H3K36 is associated with active chromatin; H3K36 trimethylation is required for homologous recombination repair and genome stability, which depends on the methyltransferase function of SETD2 (23). Haploinsufficiency of the SETD2 gene has been shown to drive genomic instability in the early phase of RCC (24). SETD2 loss-of-function also promotes renal cancer branched evolution through DNA repair impairment and replication stress (25). In the present cohort, the rate of SETD2 mutations was higher compared with that reported in the COSMIC/TCGA database. Therefore, further downstream validation of the role of SETD2 in ccRCC within Taiwanese patients is necessary.

The BAPl gene encodes the deubiquitinating enzyme BRCA1-associated protein-1, which acts with other co-factors to epigenetically regulate genes targeted by polycomb repressive complex 1, regulates gene transcription and deubiquitylates target substrates, such as BRCA1-associated RING domain 1, ubiquitylation of histone $2 \mathrm{~A}$ and $\mathrm{O}$-glucosyltransferase (26). In RCC, a mutation in BAPl causes disruption of the host cell factor-1 binding motif of BAP1 and impairs BAP1-mediated suppression of cell proliferation (27). Notably, BAPl loss is mutually exclusive with $P B R M 1$ in the literature $(4,8)$. In the present cohort, there were 25 (26.04\%) patients with $P B R M 1$ mutation and 9 (9.37\%) with BAPl mutation(s), but only one (1.04\%) patient had both $P B R M 1$ and $B A P 1$ mutations (data not shown), which is compatible to the literature. In addition, the mutation of SETD2 and BAP1 in the present Taiwanese cohort also showed mutually exclusive trend. None of the 96 patients had both SETD2 and BAP1 mutation. Mutual exclusive mutations are important to develop synthetic lethality therapies (28). Further studies to investigate the interaction of these genes may discover the potential therapeutic role for ccRCC.

The genetic landscapes of several cancer types have been described through the development of high-throughput sequencing technology $(4,8)$. Thereafter, the genetic biomarkers that can be used to predict the survival outcomes of patients with specific cancer types have been widely investigated. VHL is the key driver gene in $~ 50 \%$ of patients with ccRCC. However, a recently published meta-analysis showed that VHL mutation status is not associated with clinicopathological parameters, such as nuclear grade, disease stage or OS (29). Nevertheless, a specific type of $V H L$ dysregulation, such as $V H L$ methylation combined with other VHL pathway-associated markers like HIF1- $\alpha$ and ERK5 protein, can help in predicting disease-specific survival for all stages of ccRCC (30). The results from various studies evaluating the prognostic value of $P R B M 1, B A P 1$ and SETD2 are inconsistent. The lack of expression of PBRM1 has been shown to be associated with poor recurrence-free as well as cancer-specific survival $(31,32)$. BAP1 expression has been associated with high Fuhrman grade, advanced pathological Tumor stage, sarcomatoid dedifferentiation and significantly worsened disease-free survival and OS for patients with non-metastatic ccRCC (33). However, another study revealed that BAP1 and SETD2 mutations are associated with decreased cancer-specific survival (CSS), but the same was not true of PBRM1 for all stages of ccRCC (34). Furthermore, one study employing an immunohistochemistry microarray to evaluate the association between different markers with OS, CSS and progression-free survival (PFS) for localized ccRCC showed that there was no association with BAP1 and PBRM1 expression (35). Another study integrated recurrent somatic mutations with clinical outcomes for $>1,000$ patients with ccRCC at varying cancer stages and reported that $B A P I$ mutation is associated with large tumor size, TP53 mutation is associated with poor CSS and SETD2 mutation is associated with poor PFS (36). The present data indicated that $V H L$, $P B R M 1, S E T D 2$ and $B A P 1$ are not associated with OS in all stages of ccRCC. The diverse conclusions of these genetic prognostic biomarkers indicate that pathogenesis and cancer progression are associated with multiple gene dysregulations and that a single gene mutation is less likely to be a strong predictor.

Notably, half of the top eight highly mutated genes are epigenetic modifiers (SETD2, PBRM1, BAP1 and KDM5C). Indeed, as more techniques for epigenomic studies are quickly developed, comprehensive genomic and epigenomic studies are being introduced. A comprehensive molecular characterization of RCC using TCGA database published in 2018 demonstrated that somatic alteration of $B A P 1, P B R M 1$ and metabolic pathways correlates with subtype-specific decreased survival, and cyclin-dependent kinase inhibitor 2A alteration, DNA hypermethylation and $\mathrm{T}$ helper 2 immune signature are correlated with decreased survival within all subtypes of RCC (37). As the chromatin accessibility landscape of numerous types of primary human cancer is developed (38), further investigation of the genomic and epigenomic interactions and improved understanding of the fundamental regulatory basis of carcinogenesis is expected. 
In the present study only 12 patients had metastatic disease who received TKIs as systemic treatments. This number was not sufficient to analyze the association of gene mutations with response to the systemic treatments. However, in the recent reports published by the National Health Insurance Administration of Taiwan, the response rate $(\sim 35 \%)$ of Taiwanese patients with RCC to immune checkpoint inhibitors was higher compared with patients in the clinical trials in Western countries (39). Further comprehensive genetic and epigenetic studies as well as gene expression and downstream validation are necessary to resolve the possible mechanisms underlying these differences.

There were some limitations to the present study. First, paired normal tissues were not sequenced as controls. Thus, copy number alteration and deep analysis could not be performed. Second, targeted sequencing was used, and only the top eight genes associated with ccRCC in COSMIC were included. In this manner, some potential unique gene alterations in the Taiwanese cohort might have been missed. Third, downstream validation of each mutated gene was not performed due to insufficient remaining tissue material. Therefore, the expression changes of affected protein(s) and association with clinicopathological parameters could not be evaluated. However, most of the mutations of the target genes were non-synonymous mutations that would cause the alteration of protein expression. At last, the mean follow-up time was not long enough, and subsequent adjuvant or systemic treatments were not evaluated. This may have affected the results of gene mutation impact on survival. Nevertheless, the present study still provided information concerning the commonly mutated gene status associated with ccRCC in a Taiwanese cohort.

Overall, the current data showed that the highly frequently mutated genes associated with ccRCC in Taiwan are similar to those reported in the COSMIC/TCGA databases. However, the concurrence of $V H L$ and $P B R M 1$ mutation was $\leq 20 \%$ in the present cohort, and the SETD2 mutation rate was also higher compared with the COSMIC/TCGA cohorts. SETD2 mutation was mutually exclusive to $B A P 1$ mutation, in addition to $P B R M 1$. These results indicated that role of SETD2 mutation may be distinct in Taiwanese cohort. Further comprehensive genetic and epigenetic studies such as somatic mutations, DNA methylation assay, chromatin immunoprecipitation sequencing, assay for Transposase-Accessible Chromatin using sequencing as well as downstream validations with gene expression and functional study are necessary to validate the function and interaction of these somatic mutations.

\section{Acknowledgments}

The authors would like to thank Mr. Yu-Sin Chang and Dr Chin-Hsuan Hsieh (Lab of Uro-Oncology, Chang Gung Memorial Hospital) for their assistance.

\section{Funding}

This study was supported by The Chang Gung Medical Research Program (grant nos. CORPG3F0291, CMRPG3E1941-2 and CORPG3J0121).

\section{Availability of data and materials}

The datasets used and analyzed during the current study are available from the corresponding author on reasonable request. The additional datasets analyzed during the current study are available in the COSMIC and TCGA database.

\section{Authors' contributions}

PHL conducted the study design, analyzed and interpretated the data and wrote the manuscript. CYH conducted the study design and analyzed the data. KJY and HCK did the DNA extraction and performed the targeted sequencing. YCL collected the samples and extracted the DNA. CKC and CYL collected and processed the samples. YHC and IHS assisted to collect and process the samples and managed the administrative work and funds. STP conceived and designed the study and revised the manuscript. All authors read and approved the final manuscript.

\section{Ethics approval and consent to participate}

The study was approved by the Institutional Review Board of Chang Gung Memorial Hospital (approval no. 106-3050C) and National Taiwan University Hospital (approval no. 201312158RIND). Written informed consent was provided by all patients.

\section{Patient consent for publication}

Not applicable.

\section{Competing interests}

The authors declare that they have no competing interests.

\section{References}

1. Siegel RL, Miller K and Jemal A: Cancer statistics, 2019. CA Cancer J Clin 69: 7-34, 2018.

2. 2016 Taiwan CancerRegistry.https://www.hpa.gov.tw/Pages/Detail aspx?nodeid=269\&pid=10227. Accessed April 4, 2020 .

3. Shuch B, Amin A, Armstrong AJ, Eble JN, Ficarra V, Lopez-Beltran A, Martignoni G, Rini BI and Kutikov A: Understanding pathologic variants of renal cell carcinoma: Distilling therapeutic opportunities from biologic complexity. Eur Urol 67: 85-97, 2015.

4. COSMIC Cancer Browser. https://cancer.sanger.ac.uk/cosmic/ browse/tissue?wgs=off\&sn=kidney\&ss=NS\&hn=carcinoma\&sh=clear cell_renal_cell_carcinoma\&in $=t \& s r c=$ tissue \&all_data $=$. Accessed February 15, 2020.

5. Motzer RJ, Hutson TE, Tomczak P, Michaelson MD, Bukowski RM, Rixe O, Oudard S, Negrier S, Szczylik C, Kim ST, et al: Sunitinib versus interferon alfa in metastatic renal-cell carcinoma. N Engl J Med 356: 115-124, 2007.

6. Kim WY and Kaelin WG: Role of VHL gene mutation in human cancer. J Clin Oncol 15: 4991-5004, 2004.

7. Xing $\mathrm{T}$ and $\mathrm{He} \mathrm{H}$ : Epigenomics of clear cell renal cell carcinoma: Mechanisms and potential use in molecular pathology. Chin J Cancer Res 28: 80-91, 2016.

8. Cancer Genome Atlas Research Network: Comprehensive molecular characterization of clear cell renal cell carcinoma. Nature 499: 43-49, 2013.

9. Edge SB and Compton CC: The American joint committee on cancer: The 7th edition of the AJCC cancer staging manual and the future of TNM. Ann Surg Oncol 17: 1471-1474, 2010.

10. Xu C, Ranjbar MR, Wu Z, DiCarlo J and Wang Y: Detecting very low allele fraction variants using targeted DNA sequencing and a novel molecular barcode-aware variant caller. BMC Genomics 18: 5, 2017. 
11. Li H and Durbin R: Fast and accurate short read alignment with burrows-wheeler transform. Bioinformatics 25: 1754-1760, 2009.

12. McKenna A, Hanna M, Banks E, Sivachenko A, Cibulskis K, Kernytsky A, Garimella K, Altshuler D, Gabriel S, Daly M and DePristo MA: The genome analysis toolkit: A mapReduce framework for analyzing next-generation DNA sequencing data. Genome Res 20: 1297-1303, 2010.

13. Cibulskis K, Lawrence MS, Carter SL, Sivachenko A, Jaffe D, Sougnez C, Gabriel S, Meyerson M, Lander ES and Getz G: Sensitive detection of somatic point mutations in impure and heterogeneous cancer samples. Nat Biotechnol 31: 213-219, 2013.

14. McLaren W, Pritchard B, Rios D, Chen Y, Flicek P and Cunningham F: Deriving the consequences of genomic variants with the ensembl API and SNP effect predictor. Bioinformatics 26: 2069-2070, 2010.

15. Capitanio U, Bensalah K, Bex A, Boorjian SA, Bray F, Coleman J, Gore JL, Sun M, Wood C and Russo P: Epidemiology of renal cell carcinoma. Eur Urol 75: 74-84, 2019.

16. Brugarolas J: Molecular genetics of clear-cell renal cell carcinoma. J Clin Oncol 32: 1968-1976, 2014.

17. Kaelin WG Jr: The von hippel-lindau tumor suppressor protein and clear cell renal carcinoma. Clin Cancer Res 13: 680s-684s, 2007.

18. Posadas EM, Limvorasak S and Figlin RA: Targeted therapies for renal cell carcinoma. Nat Rev Nephrol 13: 496-511, 2017.

19. Kondo K, Yao M, Yoshida M, Kishida T, Shuin T, Miura T, Moriyama M, Kobayashi K, Sakai N, Kaneko S, et al: Comprehensive mutational analysis of the VHL gene in sporadic renal cell carcinoma: Relationship to clinicopathological parameters. Genes Chromosomes Cancer 34: 58-68, 2002.

20. Hodges C, Kirkland JG and Crabtree GR: The many roles of $\mathrm{BAF}(\mathrm{mSWI} / \mathrm{SNF})$ and PBAF complexes in cancer. Cold Spring Harb Perspect Med 6: pii: a026930, 2016.

21. Varela I, Tarpey P, Raine K, Huang D, Ong CK, Stephens P, Davies H, Jones D, Lin ML, Teague J, et al: Exome sequencing identifies frequent mutation of the SWI/SNF complex gene PBRM1 in renal carcinoma. Nature 469: 539-542, 2011.

22. Nargund AM, Pham CG, Dong Y, Wang PI, Osmangeyoglu HU, Xie Y, Aras O, Han S, Oyama T, Takeda S, et al: The SWI/SNF protein PBRM1 restrains VHL-loss-driven clear cell renal cell carcinoma. Cell Rep 18: 2893-2906, 2017.

23. Pfister SX, Ahrabi S, Zalmas LP, Sarkar S, Aymard F, Bachrati CZ, Helleday T, Legube G, La Thangue NB, Porter AC and Humphrey TC: SETD2-Dependent histone H3K36 trimethylation is required for homologous recombination repair and genome stability. Cell Rep 7: 2006-2018, 2014.

24. Chiang YC, Park IY, Terzo EA, Tripathi DN, Mason FM, Fahey CC, Karki M, Shuster CB, Sohn BH, Chowdhury P, et al: SETD2 haploinsufficiency for microtubule methylation is an early driver of genomic instability in renal cell carcinoma. Cancer Res 78: 3135-3146, 2018.

25. Kanu N, Gronroos E, Martinez P, Burrell RA, Yi Goh X, Bartkova J, Maya-Mendoza A, Mistrík M, Rowan AJ, Patel $\mathrm{H}$, et al: SETD2 loss-of-function promotes renal cancer branched evolution through replication stress and impaired DNA repair. Oncogene 34: 5699-5708, 2015.

26. Carbone M, Yang H, Pass HI, Krausz T, Testa JR and Gaudino G: BAP1 and cancer. Nat Rev Cancer 13: 153-159, 2013.

27. Peña-Llopis S, Vega-Rubín-de-Celis S, Liao A, Leng N, Pavía-Jiménez A, Wang S, Yamasaki T, Zhrebker L, Sivanand S, Spence P, et al: BAP1 loss defines a new class of renal cell carcinoma. Nat Genet 44: 751-759, 2012.
28. Huang A, Garraway LA, Ashworth A and Weber B: Synthetic lethality as an engine for cancer drug target discovery. Nat Rev Drug Discov 19: 23-38, 2020.

29. Kim HS, Kim JH, Jang HJ, Han B and Zang DY: Clinicopathologic significance of VHL gene alteration in clear-cell renal cell carcinoma: An updated meta-analysis and review. Int J Mol Sci 19 E2529, 2018.

30. Salinas-Sanchez AS, Serrano-Oviedo L, Nam-Cha SY, Roche-Losada O, Sánchez-Prieto R and Giménez-Bachs JM: Prognostic value of the VHL, HIF-1 $\alpha$, and VEGF signaling pathway and associated MAPK (ERK1/2 and ERK5) pathways in clear-cell renal cell carcinoma. A long-term study. Clin Genitourin Cancer 15: e923-e33, 2017.

31. da Costa WH, Rezende M, Carneiro FC, Rocha RM, da Cunha IW, Carraro DM, Guimaraes GC and de Cassio Zequi S: Polybromo-1 (PBRM1), a SWI/SNF complex subunit is a prognostic marker in clear cell renal cell carcinoma. BJU Int 113: E157-E163, 2014.

32. Pawlowski R, Muhl SM, Sulser T, Krek W, Moch H and Schraml P: Loss of PBRM1 expression is associated with renal cell carcinoma progression. Int J Cancer 132: E11-E117, 2013.

33. Kapur P, Christie A, Raman JD, Then MT, Nuhn P, Buchner A, Bastian P, Seitz C, Shariat SF, Bensalah K, et al: BAP1 immunohistochemistry predicts outcomes in a multi-institutional cohort with clear cell renal cell carcinoma. J Urol 191: 603-610, 2014.

34. Hakimi AA, Ostrovnaya I, Reva B, Schultz N, Chen YB, Gonen M, Liu H, Takeda S, Voss MH, Tickoo SK, et al: Adverse outcomes in clear cell renal cell carcinoma with mutations of 3p21 epigenetic regulators BAP1 and SETD2: A report by MSKCC and the KIRC TCGA research network. Clin Cancer Res 19: 3259-3267, 2013.

35. Kim SH, Park WS, Park EY, Park B, Joo J, Joung JY, Seo HK, Lee KH and Chung J: The prognostic value of BAP1, PBRM1, pS6, PTEN, TGase2, PD-L1, CA9, PSMA, and Ki-67 tissue markers in localized renal cell carcinoma: A retrospective study of tissue microarrays using immunohistochemistry. PLoS One 12: e0179610, 2017.

36. Manley BJ, Zabor EC, Casuscelli J, Tennenbaum DM, Redzematovic A, Becerra MF, Benfante N, Sato Y, Morikawa T, Kume $\mathrm{H}$, et al: Integration of recurrent somatic mutations with clinical outcomes: A pooled analysis of 1049 patients with clear cell renal cell carcinoma. Eur Urol Focus 3: 421-427, 2017.

37. Ricketts CJ, De Cubas AA, Fan H, Smith CC, Lang M, Reznik ED, Bowlby R, Gibb EA, Akbani R, Beroukhim R, et al: The cancer genome atlas comprehensive molecular characterization of renal cell carcinoma. Cell Rep 23: 313-326, 2018

38. Corces MR, Granja JM, Shams S, Louie BH, Seoane JA, Zhou W, Silva TC, Groeneveld C, Wong CK, Cho SW, et al: The chromatin accessibility landscape of primary human cancers. Science 362: eaav1898, 2018.

39. Data published by the Taiwanese National Health Insurance Administration. https://www.nhi.gov.tw/Content_List.aspx?n=71 57A9A3E2A3B110\&topn=3FC7D09599D25979. Accessed April 4,2020 .

This work is licensed under a Creative Commons Attribution-NonCommercial-NoDerivatives 4.0 International (CC BY-NC-ND 4.0) License. 